\title{
BER Performance Analysis for Optical Communication using DPSK Modulation
}

\author{
Md. Mobarok Hossain Rubel \\ Lecturer, \\ Dept. of Electrical and Electronic Engineering \\ Leading University, Sylhet.
}

\author{
Syed Golam Mahmud \\ Lecturer, \\ Dept. of Electrical and Electronic Engineering \\ Leading University, Sylhet.
}

\begin{abstract}
In this paper we study and analyze Bit Error Rate (BER) performance for optical communication system using Differential Phase Shift Keying (DPSK) modulation scheme. Specifically, after developing an approximate form BER expression, we study the BER performance for different system parameters such as fiber length, gain and number of amplifier. With the aid of analysis and simulation results we demonstrate the influence of different system parameters on the BER performance of an optical system using differential modulation schemes.
\end{abstract}

Keywords- Optical Communication, BER, DPSK, Fiber Length, Bit Rate, Fiber Gain.

Major Area- Communication and Network, Signal Processing for Communications, Optical Fiber Communication.

\section{INTRODUCTION}

Recently Differential Phase Shift Keying (DPSK) has been proved to be well suited for high spectral density transmission even at transoceanic distances [6, 13]. This modulation technique also exhibits a large tolerance to inline cumulated dispersion for distances up to $13000 \mathrm{~km} \mathrm{[2,8].} \mathrm{Considering}$ these two key characteristics we can assume that DPSK might be a good candidate for upgrading the already installed submarine links [11]. Usually, typical lines are made by nondispersion flattened fibers where dispersion, ranging from $6000 \mathrm{ps} / \mathrm{nm}$ to $+6000 \mathrm{ps} / \mathrm{nm}$, can be observed over the whole C-band for a $5000 \mathrm{~km}$ link [2, 9]. This aspect is fundamentally different from another $40 \mathrm{~Gb} / \mathrm{s}$ Return Zero (RZ)-DPSK transmission over Non-zero-dispersion-shifted-fibers (NZDSF) where Raman amplification was used along with a special arrangement of dispersion compensation modules to keep the cumulated dispersion below $1500 \mathrm{ps} / \mathrm{nm}$ [4].
As shown in [4 and 12], in differential phase shift keying (DPSK), it is possible to apply differential modulation either in the time or frequency domain depending on the condition of fading channels. However, the BER performance of those systems is influenced by time or frequency correlation between adjacent symbols. Considering these factors many theoretical analysis have been done on BER performance degradation. In addition to these factors, BER performance of optical communication system is also constrained by few other parameters such as fiber length, amplifier gain, bit rate etc. Nevertheless, considering the parameters, development of a closed form expression of the BER for optical fiber communication system has not been given much attention. But it is important to have a quantitative understanding of the influence of other system parameters upon the BER performance [9]. The transmission system considered in [14] is based on conventional transmitter and receiver operating at $1550 \mathrm{~nm}$ wavelength with single mode fiber. The quality of detected data is improved by reducing embedded noise induced by linear and nonlinear effects of optical transmission channel. In [15], experimental evaluation of Praseodymium Doped Fiber Amplifier (PDFA) has been shown based on BER measurements. The study also presents a booster amplifier, configured in a co-propagating scheme, as a function of both wavelength and optical input power. In addition to the above, [16] presents some analysis and derivation of the BER in an optical transmission system with different types of optical amplifiers such that amplifiers add Gaussian spontaneous emission noise which dominates over other sources of noise.

In this paper we show an approximate closed form expression of the BER in DPSK systems over optical channel taking account of the differential modulation in both time and

frequency domains. More specifically, we show the BER performance considering few optical parameters such as fiber length, gain and number of amplifiers used in a typical optical transmission system. 


\section{SYSTEM MODEL}

\section{A) Block Diagram and Signal Model}

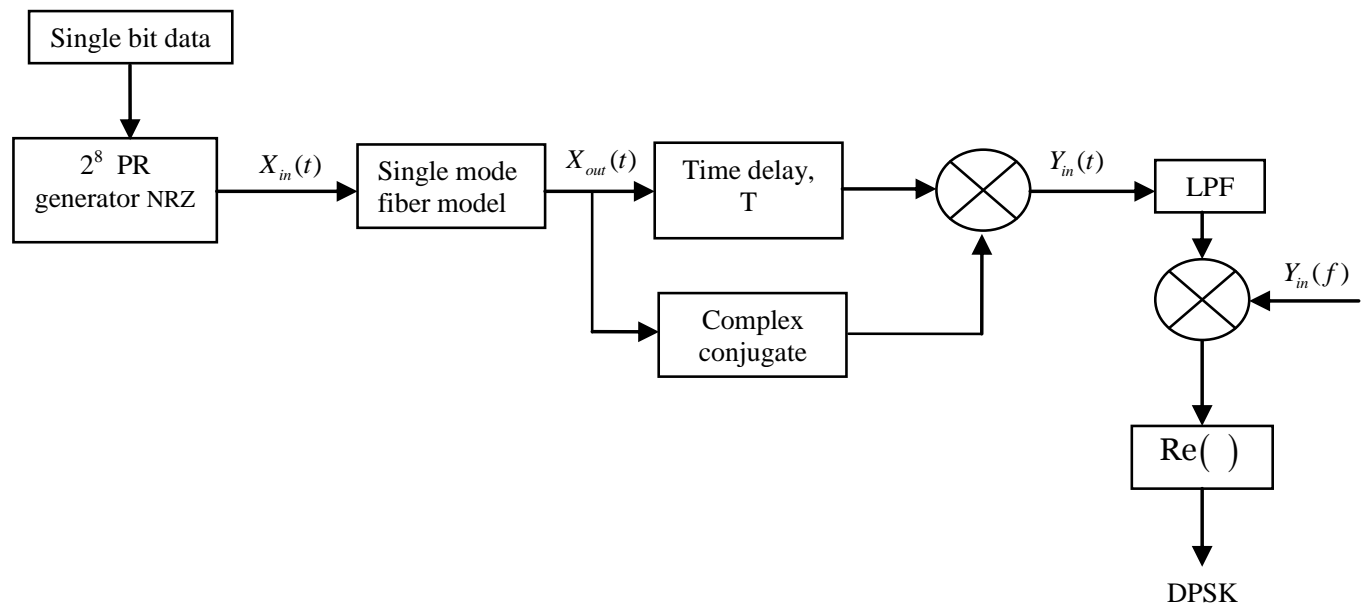

Fig. 1 Block diagram for DPSK transmission system

In the Fig. 1 block diagram of DPSK transmission system is shown. In this block a single bit data is transmitted through single-mode fiber. Before transmitting the optical source is assumed to be a single frequency laser with linewidth significantly less than the modulation rate, so that the effect of the laser phase noise is negligible. The laser is modulated according to a pseudorandom (PR) code of length $2^{8}$ with a non-return-zero (NRZ) input data signal so that Inter Symbol Interference (ISI) due to chromatic dispersion can be accurately simulated [8]. The bit is shifted in frequency domain by discrete Fourier transform. The single-mode fiber is modelled as a band pass filter with flat amplitude response

and linear group delay within the data bandwidth. These are valid assumptions, since the transmission systems discussed in this paper have narrow bandwidths with respect to the absolute value of the optical frequency $[7,8]$.

The output of the PR code is used to generate the signals for the DPSK systems. The complex equivalent lowpass modulated signals for DPSK at the fiber input is sent.

For the DPSK and systems the modulated signals at the fiber input are: [8]

$$
X_{i n}(t)=\sum_{k} a_{k} P(t-k T)
$$

where $a= \pm 1$ for PSK or DPSK, $k$ is an integer, $T$ is the bit time and $P(t)$ is the pulse function with unity amplitude within the bit time. A single bit data is transformed into frequency domain by discrete Fourier transform.

\section{B) Fiber transfer function}

The single-mode fiber is modelled as a bandpass filter with flat amplitude response and linear group delay within the data bandwidth. The low-pass equivalent model for the singlemode fiber transfer function is then given by

$$
\begin{aligned}
& H(f)=e^{-j \Psi(f)}=e^{-j f^{2}} \\
= & e^{-j}\left[\alpha B^{2}(f / B)^{2}\right],
\end{aligned}
$$

where $\quad \alpha=\pi D(\lambda)^{\frac{\lambda^{2}}{c}} L$,

$f$ is the difference between the optical frequency and optical carrier frequency, $B$ is the data rate or bit rate, $\lambda$ is the operating wavelength, $c$ is the speed of light, $D$ is the dispersion factor and $L$ is the fiber length.

The signal at the output of the fiber $X_{\text {out }}(t)$ is given by

$X_{\text {out }}(f)=X_{\text {in }}(f) H(f)$,

where $X_{\text {out }}(f)$ and $X_{\text {in }}(f)$ are the Fourier transform of output and input symbol respectively and can be given by

$$
X_{\text {out }}(f)=\int_{-\alpha}^{\alpha} X_{\text {out }}(t) e^{-j 2 \pi f t} d t
$$

and

$X_{i n}(f)=\int_{-\alpha}^{\alpha} X_{i n}(t) e^{-j 2 \pi f t} d t$

From the block diagram in Fig. 1, $Y_{i n}(t)$ can be given by $Y_{\text {in }}(t)=\left[\begin{array}{c}\mathrm{I}_{m}\left\{X_{\text {out }}(t)\right\} \operatorname{Re}\left\{X_{\text {out }}(t-T)\right\} \\ -\operatorname{Re}\left\{X_{\text {out }}(t)\right\} I_{m}\left\{X_{\text {out }}(t-T)\right\}\end{array}\right]$

where $\operatorname{Re}\{\}$ and $\operatorname{Im}\{\}$ denote the real part and the imaginary part (in terms of low-pass representation) respectively.

In the DPSK system, the signal at the fiber output is differentially detected (delayed by one bit and then multiplied) and then processed by the baseband filter. The baseband filter input $Y_{i n}(t)$ is given by

$$
Y_{\text {in }}(t)=\left[\begin{array}{l}
\operatorname{Re}\left\{X_{\text {out }}(t)\right\} \operatorname{Re}\left\{X_{\text {out }}(t-T)\right\} \\
+I_{m}\left\{X_{\text {out }}(t)\right\} I_{m}\left\{X_{\text {out }}(t-T)\right\}
\end{array}\right]
$$


where $T$ is the time delay which is always inverse of the bit rate $[1,8]$. Note that, for a proper transmission channel, if the bandwidth of the channel is $B$ then the bit rate will be equal to $2 B$. So, we have another relation between time delay and bandwidth as

$\frac{T}{2}=\frac{1}{2 \text { Bit rate }}=\frac{1}{\text { Bandwidth }}$

Consequently, we assume the electrical signal delay, $T_{e}$ has a relation with the electrical bandwidth $B_{e}$ according to $T_{e}=\frac{2}{B_{e}}$. Note that, using Fourier transform the signal $Y_{\text {in }}(t)$ converted into frequency domain $Y_{i n}(f)$ multiplied with the low pass filter transfer function. Here Butterworth filter is used.

The frequency response of the Butterworth filter is maximally flat (has no ripples) in the passband, and rolls off towards zero in the stopband. The filter transfer function is given by [7]

$\left|H_{n}(f)\right|=\sqrt{\frac{1}{1+\left(f / f_{c}\right)^{2 n}}}$,

where $f_{c}$ is the upper $3 \mathrm{~dB}$ cut off point and $n$ represents the order of the filter. Then one bit is shifted multiplied with transmitted bit. By discrete Fourier transform a frequency response is obtained as $Y_{i n}(f)$. And it multiplied with the Butterworth low pass filter with order $n=5$. From this finally the single bit data is received [7]. $Y_{\text {out }}(f)=\left|H_{n}(f)\right| Y_{\text {in }}(f)$

Finally, the received DPSK output signal $Y_{\text {out }}(t)$ is obtained by inverse Fourier transform of $Y_{\text {out }}(f)$.

\section{BER CALCULATION}

The signal power received after photo detection by a P-i$\mathrm{N}$ photo detector can be given by,

$$
P_{s}=\left(R P_{i n}\right)^{2}
$$

where $R$ is the responsivity [2] of the photodiode, $P_{i n}$ is the input power given by $P_{i n}=10^{\frac{P_{S}}{10}}$ with $P_{S}$ being the signal power. The shot noise induced in $\mathrm{P}-\mathrm{i}-\mathrm{N}$ photo detector can be given by

$$
N_{\text {shot }}=2 B_{e} \eta_{\text {out }} L\left(G I_{s} \eta_{\text {in }}+I_{s p}\right),
$$

where $B_{e}$ is the electrical bandwidth $[7,9], \eta_{\text {out }}$ and $\eta_{\text {in }}$ are the amplifier output and input coupling efficiency respectively, $G$ is the optical gain, $I_{S}$ and $I_{s p}$ are the PCE of amplifier input power and spontaneous emission power respectively.
As shown in [6], $I_{s}$ can be given by

$$
I_{s}=R I_{i n} G L,
$$

and $I_{s p}$ can given by

$$
I_{s p}=N_{s p}(G-1) e B_{0},
$$

where $N_{s p}=1$ for an ideal amplifier and can range from 1.4 to 4 depending both on the pumping rate and the operating wavelength and $B_{0}$ is the optical bandwidth, $e$ is the electron charge. Thermal noise is given by

$$
N_{t h}=\left(I_{t h}\right)^{2},
$$

where $I_{t h}$ is the thermal current. Signal-spontaneous beat noise is given by

$$
N_{s-s p}=4 G_{s} I_{s} \eta_{\text {in }} \eta_{\text {out }}{ }^{2} L^{2} B_{e} / B_{0}
$$

where $G_{S}$ is gain of the amplifier $[2,6]$.

Now the frequency domain input signal at the receiver can be given by

$$
Y_{i n}(f)=\int_{-\alpha}^{\alpha} Y_{i n}(t) e^{-j 2 \pi f t} d t
$$

From (8) we put the value of $Y_{i n}(t)$ so that (18) becomes

$Y_{\text {in }}(f)=\int_{-\alpha}^{\alpha}\left[\begin{array}{c}\operatorname{Re}\left\{X_{\text {out }}(t)\right\} \operatorname{Re}\left\{X_{\text {out }}\left(t-T_{e}\right)\right\} \\ +\operatorname{Im}\left\{X_{\text {out }}(t)\right\} \operatorname{Im}\left\{X_{\text {out }}\left(t-T_{e}\right)\right\}\end{array}\right] e^{-j 2 \pi f t} d t$

$$
\begin{aligned}
& =\int_{-\alpha}^{\alpha}\left[\left\{X_{\text {out }}(t) X_{\text {out }}\left(t-T_{e}\right)\right\} \cos \left\{X_{\text {out }}(t) X_{\text {out }}\left(t-T_{e}\right)\right\} e^{-j 2 \pi f t}\right] d t \\
& -j \int_{-\alpha}^{\alpha}\left[\left\{X_{\text {out }}(t) X_{\text {out }}\left(t-T_{e}\right)\right\} \sin \left\{X_{\text {out }}(t) X_{\text {out }}\left(t-T_{e}\right)\right\} e^{-j 2 \pi f t}\right] d t \\
& +j \int_{-\alpha}^{\alpha}\left[\left\{X_{\text {out }}(t) X_{\text {out }}\left(t-T_{e}\right)\right\} \cos \left\{X_{\text {out }}(t) X_{\text {out }}\left(t-T_{e}\right)\right\} e^{-j 2 \pi f t}\right] d t \\
& -j \int_{-\alpha}^{2}\left[\left\{X_{\text {out }}(t) X_{\text {out }}\left(t-T_{e}\right)\right\} \sin \left\{X_{\text {out }}(t) X_{\text {out }}\left(t-T_{e}\right)\right\} e^{-j 2 \pi f t}\right] d t
\end{aligned}
$$

As shown in [7], after some calculations, (20) can be written as

$$
Y_{\text {in }}(f)=\left[\begin{array}{c}
(1+j) \cos \left\{X_{\text {out }}\left(\delta-T_{e}\right) X_{\text {out }}\left(\delta+T_{e}\right)\right\} \\
+(1-j) \sin \left\{X_{\text {out }}(\delta-T) X_{\text {out }}\left(\delta+T_{e}\right)\right\}
\end{array}\right]
$$

Now, the frequency domain output signal from the fiber can be expressed as 


$$
\begin{gathered}
Y_{\text {out }}(f)=\left|H_{n}(f)\right| Y_{\text {in }}(f) \\
=\left|H_{n}(f)\right|\left[\begin{array}{l}
(1+j) \cos \left\{X_{\text {out }}\left(\delta-T_{e}\right) X_{\text {out }}\left(\delta+T_{e}\right)\right\} \\
+(1-j) \sin \left\{X_{\text {out }}\left(\delta-T_{e}\right) X_{\text {out }}\left(\delta+T_{e}\right)\right\}
\end{array}\right]
\end{gathered}
$$

The received signal power can be obtained

$$
\begin{gathered}
\left.Y_{\text {out }}(f) Y_{\text {out }}{ }^{*}(f)=\left|H_{n}(f)\right| \begin{array}{l}
(1+j) \cos \left\{X_{\text {out }}\left(\delta-T_{e}\right) X_{\text {out }}\left(\delta+T_{e}\right)\right\} \\
+(1-j) \sin \left\{X_{\text {out }}\left(\delta-T_{e}\right) X_{\text {out }}\left(\delta+T_{e}\right)\right\}
\end{array}\right] \\
\times\left|H_{n} *(f)\right|\left[\begin{array}{l}
(1-j) \cos \left\{X_{\text {out }}^{*}\left(\delta-T_{e}\right) X_{\text {out }}^{*}\left(\delta+T_{e}\right)\right\} \\
+(1+j) \sin \left\{X_{\text {out }}^{*}\left(\delta-T_{e}\right) X_{\text {out }}^{*}\left(\delta+T_{e}\right)\right\}
\end{array}\right] \\
=2\left[1+\sin \left(\operatorname{Re}\left[X_{\text {out }}\left(\delta-T_{e}\right) X_{\text {out }}\left(\delta+T_{e}\right)\right]\right)\right]
\end{gathered}
$$

where $T_{e}=\frac{4 \eta_{\text {out }} L\left(G I_{s} \eta_{\text {in }}+I_{s p}\right)}{N_{\text {shot }}}$. From (24) it can be seen that, the fiber output is greatly affected by both shot noise and also by the other noise parameters. Similar calculation can be shown for spontaneous-spontaneous bit noise. It can be realized that the total noise is affected by these two significant parameters. As the total noise consists of shot noise, spontaneous-spontaneous bit noise and other noises, individual noise will have their affect on calculated received signal power. Because, whatever we say about (13), (17) or (25), one parameter is common to all of them that is electrical bandwidth. If we still look into (27), we find time delay as well as electrical bandwidth reveals the relation between the amplifier input power $I_{S}$ and the received signal power $Y_{\text {out }}(f) Y_{\text {out }}^{*}(f)$ calculated in (24).

Spontaneous-spontaneous bit noise is given by [6]

$N_{s p-s p}=\left(I_{s} \eta_{o u t} L\right)^{2} B_{e}\left(2 B_{0}-B_{e}\right) / B_{0}{ }^{2}$

Total noise induced in the received signal is given by

$N_{\text {total }}=N_{s h o t}+N_{t h}+N_{s-s p}+N_{s p-s p}$

The SNR is given by

$$
S N R=\frac{I_{S}^{2}}{N_{\text {total }}}
$$

Finally, the BER can be calculated similar to [12] as

$$
B E R=\frac{1}{2} \operatorname{erfc}\left(\frac{\sqrt{S N R}}{\sqrt{2}}\right)
$$

\section{SIMULATION RESULTS}

Following the theoretical analysis presented earlier, we present some simulation results showing BER performance for an optical system using DPSK modulation scheme. The simulation is carried out for a single mode fiber operating at $1.55 \mu \mathrm{m}$. The dispersion co-efficient, $D$ of the fiber is considered as $17 \mathrm{ps} / \mathrm{km} . \mathrm{nm}$. To evaluate the BER, SNR is calculated for a P-i-N photo detector in presence of shot noise, thermal noise, signal-spontaneous bit noise and spontaneousspontaneous bit noise. The following discussion is based on the BER performance for three key parameters of the optical transmission system.

\section{A) BER performance in different fiber length}

The BER performance as a function of received power is shown Fig. 2 for different fiber length. From the figure it is observed that the BER increases when input power to the receiver is increased. Specifically, the input power required for $\mathrm{BER}=10^{-9}$ increases with the increase of fiber length. When the fiber length, $\mathrm{L}=50 \mathrm{~km}$, the input power required to the receiver to obtain $\mathrm{BER}=10^{-8}$ is found to be $-27.65 \mathrm{~dB}$. This is evaluated to be $-27 d B_{m}$ in presence of dispersion for fiber length, $\mathrm{L}=100 \mathrm{~km}$ and $-25.8 d B_{m}$ at a length, $\mathrm{L}=150$ $\mathrm{km}$. This demonstrates that the DPSK spectrum is influenced by spectrum.

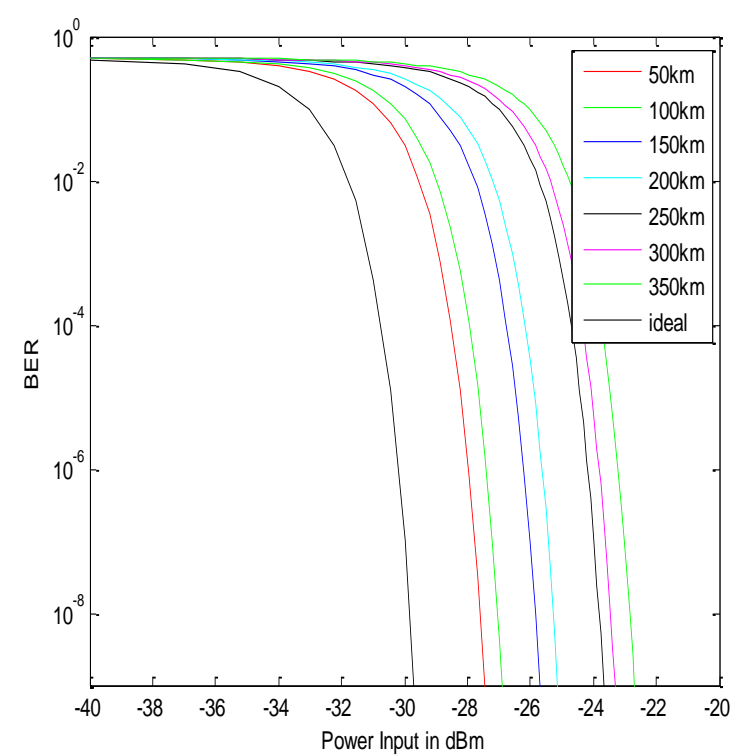

Fig. 2 BER performance of DPSK system due to variation of fiber length operating at a wavelength, $\lambda=1.55 \mu \mathrm{m}$ having dispersion co-efficient, $\mathrm{D}=17 \mathrm{ps} / \mathrm{km} . \mathrm{nm}$.

\section{B) BER performance for different number of amplifier}

We consider the most common Erbium Doped Fiber Amplifier (EDFA) pumped with a laser at a wavelength of $980 \mathrm{~nm}$ or $1480 \mathrm{~nm}$ and exhibits gain in the $1550 \mathrm{~nm}$ region [6]. In this simulation the DPSK system has been analyzed for the variation of number of amplifier used. Simulation, as shown in Fig. 3, for $\mathrm{BER}=10^{-8}$, input power required to the 
receiver is $-29.65 d B_{m}$ for $n=3,-28.6 d B_{m}$ for $n=4,-$ $26.9 \mathrm{~d} B_{m}$ for $n=5$ and $-25.55 d B_{m}$ for $n=6$. So, as the numbers of amplifier are increased, input powers required to the receiver are increased.

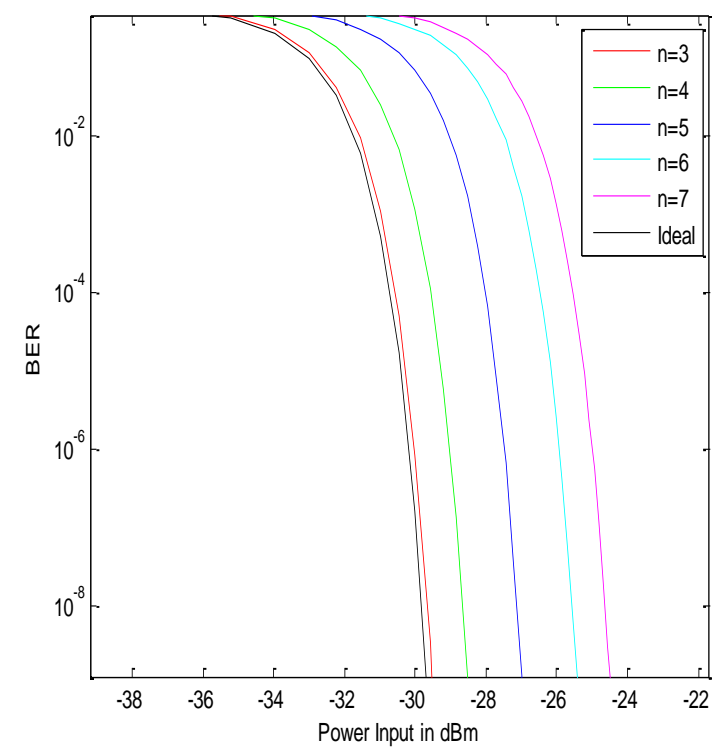

Fig. 3 BER performance of DPSK system due to variation of number of amplifier used operating at a wavelength, $\lambda=1.55$ $\mu \mathrm{m}$ having dispersion co-efficient, $\mathrm{D}=17 \mathrm{ps} / \mathrm{km} . \mathrm{nm}$.

\section{C) BER performance with different amplifier gain}

Similar to Fig. 2 the EDFA has been used in this simulation. Here BER versus input power has been shown for various amplifier gains. The result demonstrates that, with the increase of the amplifier gain, the speed of data communication increases, but the probability of error also increases i.e. BER increases. Specifically, The input power required for $\mathrm{BER}=10^{-9}$ increases with the increase in amplifier gain. When the amplifier gain, $\mathrm{Gs}=20 d B$, the input power required to the receiver to obtain $\mathrm{BER}=10^{-8}$ is found to be $-24.6 \mathrm{~d} B_{m}$. This is evaluated to be $-25.5 d B_{m}$ with amplifier gain $15 \mathrm{~dB}$ and $-22.5 \mathrm{~dB}$ for amplifier gain $25 \mathrm{~dB}$ . From figure 3.5 , it is observed that the input power required to the receiver increases with the increase in amplifier gain. This demonstrates that the DPSK spectrum is influenced by variation of amplifier gain.

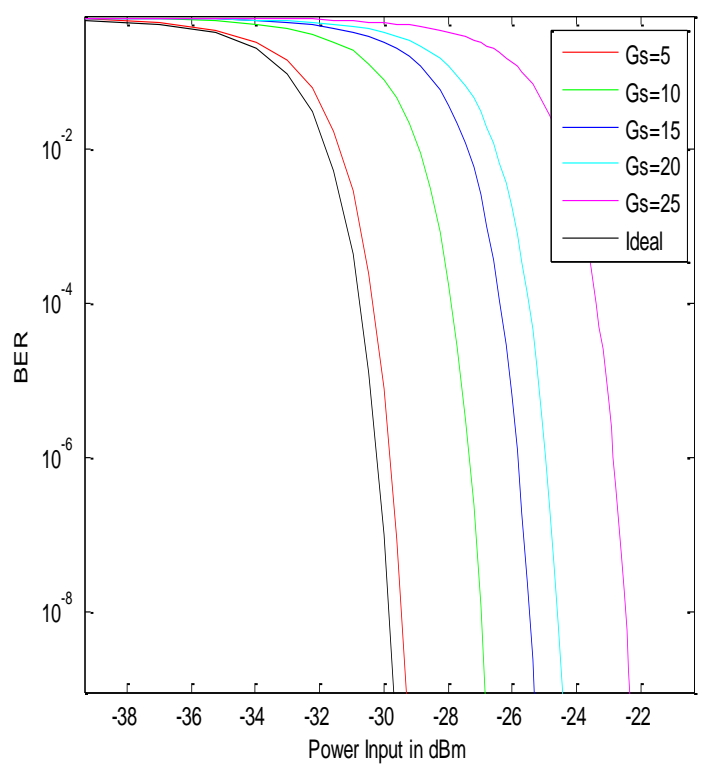

Fig. 4 BER performance of DPSK system due to variation of amplifier gain operating at a wavelength, $\lambda=1.55 \mu \mathrm{m}$ having dispersion co-efficient, $\mathrm{D}=17 \mathrm{ps} / \mathrm{km} . \mathrm{nm}$.

\section{CONCLUSION}

The performance of DPSK modulation system has been investigated in single mode fiber. The analysis is carried out in terms of BER considering fiber length, amplifier gain and number of amplifier used. In our simulations, the power penalty caused by fiber dispersion is evaluated at BER of $10^{-9}$ for a single mode fiber having dispersion co-efficient of 17 $\mathrm{ps} / \mathrm{km} . \mathrm{nm}$ at a wavelength $1.55 \mu \mathrm{m}$. To see the relative disadvantage of DPSK in comparison with PSK, consider that during some bit interval the received is so contaminated by noise that in PSK system an error would be made in the determination of whether the transmitted bit was a 1or a 0 . In DPSK a bit determination is made on the basis of the signal received in two successive bit intervals. Hence noise in one bit interval may cause errors to two bits determinations. The error rate in DPSK is therefore greater than in PSK, and it is a matter of fact, there is a tendency for bit errors to occur in pairs. It is not inevitable however those errors occur in pairs. Single errors are still possible.

\section{REFERENCES}

[1] Herbert Taub, Donald L. Schilling. Principles of Communication Systems, Tata McGraw Hill Edition, 1997, pp. 249-58.

[2] John M. Senior, Optical Fiber Communications, Eastern Economy Edition, 2008, pp. 1-4,427.

[3] Chunjun Gao; Alexandar M. Haimovich, members of IEEE :, "BER analysis of MPSK space-time block codes with differential detection" , IEEE Communications Letters, vol.7, no.7, July 2003.

[4] Fumihito Sasamori, Shiro Handa , and Shinjiro Oshita , Members , "A simple method of BER calculation in DPSK/OFDM systems over fading channels" , IEICE Trans. Fundamentals, Vol.88-A, No.1 January 2005.

[5] Jonas Hansryd, James Van Howe, Chris Xu, "Nonlinear crosstalk and compensation in QDPASK optical 
communication systems", IEEE Photonics Technology Letters, vol.16, no. 8, August 2004.

[6] Govind P. Agarwal, Fiber Optic Communication Systems, John Wiley \& sons, Inc., 2002. pp. 160-68

[7] Bernard Sklar, Digital Communications Fundamentals and Applications, Pearson Education, 2003, pp. 63-69, 151, 1023-24.

[8] Elrefaie, A.F.; Wagner, R.E.; Atlas, D.A.; Daut, D.G.; "Chromatic dispersion limitations in coherent lightwave transmission systems," Lightwave Technology, Journal of , vol.6, no.5, pp.704-709, May 1988

[9] Jin Wang; Kahn, J.M., "Impact of chromatic and polarization-mode dispersions on DPSK systems using interferometric demodulation and direct detection," Lightwave Technology, Journal of, vol.22, no.2, pp. 362- 371, Feb. 2004.

[10] Keang-Po Ho , Member IEEE , and Chinlon Lin, Fellow IEEE, "Performance analysis of optical transmission system with polarization-mode dispersion and forward error correction" , IEEE Photonics Technology Letters, Vol.9, No.9,September 1997.

[11] Keang-Po Ho, Member, IEEE, "Error probability of DPSK signals with interchannel four-wave-mixing in highly dispersive transmission systems", IEEE Photonics Technology Letters.

[12] S. P. Majumder, Member, IEEE, Afreen Azhari, and F. M. Abbou, "Impact of fiber chromatic dispersion on the BER performance of an optical CDMA IM/DD transmission system", IEEE Photonics Technology Letters, Vol. 17,No. 6, June 2005.

[13] Q. T. Zhang, Senior member, IEEE, "Exact analysis of postdetection combining for DPSK and NFSK systems over arbitrarily correlated Nakagami channels ", IEEE Transactions on Communications, Vol. 46, No. 11, November 1988.

[14] Hadj Bourdoucen, Amer Alhabsi, "Improvement of bit error rate in optical fiber receivers", World Academy of Science, Engineering and Technology, 52, 2009.

[15] R. J. W. Jonkar, R. C. Schimmel, H. de Waardt, "Experimental assessment of a Praseodymium doped fiber amplifier (PDFA) for amplifier applications in the second telecommunication window" Proceedings Symposium IEEE/LEOS Benelux Chapter,2001.

[16] Felix Abramovich, Polina Bayvel; "Some statistical remarks on the derivation of BER in amplified optical communication systems", IEEE Transactions on Communications, Vol.4, No. 9, September, 1997. 